\title{
Article \\ Effect of Nephrology Care on Mortality in Incident Dialysis Patients: A Population-Based Cohort Study
}

\author{
Cheng-Yin Chung ${ }^{1,2}$, Ping-Hsun $\mathrm{Wu}^{2,3}$, Yi-Wen Chiu ${ }^{2,4}$, Shang-Jyh Hwang ${ }^{2,4,5}$ and Ming-Yen Lin ${ }^{2,4, *(\mathbb{D})}$ \\ 1 Division of Nephrology, Department of Internal Medicine, Ministry of Health and Welfare, Pingtung Hospital, \\ Pingtung 90054, Taiwan; 950085@kmuh.org.tw \\ 2 Division of Nephrology, Department of Internal Medicine, Kaohsiung Medical University Hospital, \\ Kaohsiung Medical University, Kaohsiung 80756, Taiwan; 970392kmuh@gmail.com (P.-H.W.); \\ chiuyiwen@kmu.edu.tw (Y.-W.C.); sjhwang@kmu.edu.tw (S.-J.H.) \\ 3 Graduate Institute of Medicine, College of Medicine, Kaohsiung Medical University, \\ Kaohsiung 80756, Taiwan \\ 4 Department of Renal Care, College of Medicine, Kaohsiung Medical University, Kaohsiung 80756, Taiwan \\ 5 Institute of Population Health Sciences, National Health Research Institutes, Miaoli 35053, Taiwan \\ * Correspondence: 1080421@kmuh.org.tw; Tel.: +886-7-312-1101-7906
}

Citation: Chung, C.-Y.; Wu, P.-H.; Chiu, Y.-W.; Hwang, S.-J.; Lin, M.-Y. Effect of Nephrology Care on Mortality in Incident Dialysis Patients: A Population-Based Cohort Study. J. Pers. Med. 2021, 11, 1071. https://doi.org/10.3390/jpm11111071

Academic Editor: Marijn Speeckaert

Received: 23 September 2021

Accepted: 22 October 2021

Published: 24 October 2021

Publisher's Note: MDPI stays neutral with regard to jurisdictional claims in published maps and institutional affiliations.

Copyright: (c) 2021 by the authors. Licensee MDPI, Basel, Switzerland. This article is an open access article distributed under the terms and conditions of the Creative Commons Attribution (CC BY) license (https:/ / creativecommons.org/licenses/by/ $4.0 /)$.

\begin{abstract}
Long-term and continuous nephrology care effects on post-dialysis mortality remain unclear. This study aims to systematically explore the causal effect of nephrology care on mortality for patients with dialysis initiation. We conducted a retrospective cohort study to include incident patients with dialysis for $\geq 3$ months in Taiwan from 2004 through 2011. The continuous nephrology care of incident patients in the three years before their dialysis was measured every six months. Continuous nephrology care was determined by $0-6,0-12, \ldots, 0-36$ months and their counterparts; and none, intermittent, 0-6 months, ... , and 0-36 months. Simple and weighted hazards ratio (HR) and $95 \%$ confidence interval (CI) for one-year mortality were estimated after propensity score (PS) matching. We included a total of 44,698 patients (mean age $63.3 \pm 14.2$, male $51.9 \%$ ). Receiving $\geq 1$ year predialysis nephrology care was associated with a $22 \%$ lower post-dialysis mortality hazard. No different effects were found (ranges of PS matching HR: 0.77-0.80) when comparing the defined duration of nephrology care with their counterparts. Stepped survival benefits were newly identified in the intermittent care, which had slightly lower HRs (weighted HR: 0.88, 95\% CI: 0.79-0.97), followed by reviving care over six months to two years (ranges of weighted HR: 0.60-0.65), and reviving care over two years (ranges of weighted HR: 0.48-0.52). There was no existing critical period of nephrology care effect on post-dialysis, but there were extra survival benefits when extending nephrology care to $>2$ years, which suggests that continuous and long-term care during pre-dialysis/chronic kidney disease phase is required.
\end{abstract}

Keywords: dialysis; end-stage kidney disease; mortality; nephrology care; causal inference

\section{Introduction}

Globally, over 850 million people with chronic kidney disease (CKD) need appropriate management and care, to improve risks of adverse outcomes and elevate their quality of care [1]. Several chronic conditions usually accompany CKD and may affect consequent patient care and outcomes. For example, hypertension accounts for about $60 \%$ to $90 \%$ of the global CKD population [2], which is the main comorbidity of CKD. Diabetic nephropathy affects approximately $20 \%-40 \%$ of patients who have diabetes mellitus and also contributes to a large proportion of end-stage kidney disease (ESKD) [3]. Chronic conditions in CKD were also associated with whether patients can receive appropriate care when their disease requires it [4]. Without appropriate management and control, these chronic conditions could incur the rapid progression of renal dysfunction and attribute more risks of developing cardiovascular morbidity and mortality [5]. 
Although results from previous studies mostly support the notion that nephrology care during the pre-ESKD phase may be associated with survival benefits after dialysis is initiated [6-8], optimal nephrology care intervals, visits, and contents could be influenced by reimbursement policy and are yet to be determined. In general, timely nephrology referral, regular nephrology care visits, and multidisciplinary care were suggested when patients' glomerular filtration rate reached $30 \mathrm{~mL} / \mathrm{min} / 1.73 \mathrm{~m}^{2}$ or below, or for patients facing uncontrollable renal disease deterioration $[9,10]$. However, arbitrarily categorizing the timing of nephrology referral as early or late, and determining nephrology care in short time windows, may lose accuracy in quantifying the effects of nephrology care on patient benefits after dialysis. It is worthwhile to conduct a study to define nephrology care by longer durations before dialysis in a less restrictive reimbursement system for nephrology care.

The current study systematically determined exposure to nephrology care by sixmonthly intervals until the three years prior to dialysis, and comprehensively explored the average causal effects of nephrology care on post-dialysis mortality using the propensity score (PS) approach.

\section{Materials and Methods}

\subsection{Study Design and Population}

We conducted a retrospective population-based cohort study to assess the effect of continuous nephrology care during the pre-dialysis CKD status on one-year mortality after dialysis. All adult patients who initiated long-term ( $\geq 3$ months) dialysis therapy (hemodialysis or peritoneal dialysis) between 2007 to 2011 were identified from the Taiwan National Health Insurance Research Database, Ministry of Health and Welfare (NHIRD_MOHW). Taiwan NHIRD is a population-based claim database containing 23 million people's details of clinical information (including the date, expenditures, and diagnosis related to both inpatient and outpatient procedures; prescription details; examinations; and operations) that has been well applied in epidemiological and clinical research [11,12]. These databases are maintained and managed by Health and Welfare Data Science Center (MOHW) and have been opened to the researcher through on-site analysis with a remote connection to Taiwan's Ministry of Health and Welfare server after formal application. We identified patients who initiated long-term dialysis based on the definitions published in our previous studies ${ }^{4}$. In brief, dialysis patients were identified by several International Classification of Disease, Ninth Revision, Clinical Modification, (ICD-9-CM) codes from the Registry of Catastrophic Illness dataset and ensured those who underwent dialysis $\geq 3$ months by dialysis reimbursed codes (Supplementary Table S1 online).

\subsection{Nephrology Care}

We determined nephrology care by specialty of medical visit which could be obtained from outpatient claim data. The inspection time window of nephrology care was selected for every 6-monthly interval, retrospectively from the maintenance dialysis to three years prior. The continuous nephrology care within each 6-monthly inspection interval was defined by at least two nephrology visits, and the longest nephrology visit interval within each time-windows $\geq 3$ months. Based on the above definition, we classified the patients with or without continuous nephrology care at each setting time six-month interval and their counterparts, to determine the crucial period of nephrology care. Furthermore, we detected cumulative continuous nephrology care retrospectively from dialysis by classifying patients into the exclusive groups: none, intermittent, 0-6 months, $0-12$ months (1 year), 0-18 months, $0-24$ months, $0-30$ months, and 0-36 months. For example, patients in the $0-6$ months group were required to have at least two nephrology visits within six months before dialysis, with the longest nephrology visit interval $\geq 3$ months, to be called continuous nephrology care. Patients in the $0-12$ months group were required to have both continuous nephrology care in 0-6 and 7-12 months before dialysis, while patients in the 
$0-18$ months group were required to have continuous nephrology care in $0-6,7-12$, and 12-18 months, and so on.

\subsection{Comorbidity Assessment}

Because patients' comorbidities mainly determine patterns of nephrology care, we defined 29 comorbidities that can be obtained with high positive predicted values $(>70 \%)$ through ICD-9 CM codes from claim data [13]. The 29 comorbidities contain: atrial fibrillation, chronic heart failure, diabetes, hypertension, peripheral vascular disease, stroke or transient ischemic attack, asthma, lymphoma, metastatic cancer, non-metastatic cancer, chronic pulmonary disease, severe constipation, dementia, inflammatory bowel disease, rheumatoid arthritis, alcohol misuse, chronic pain, depression, schizophrenia, chronic viral hepatitis B, cirrhosis, epilepsy, hypothyroidism, irritable bowel syndrome, multiple sclerosis, myocardial infarction, Parkinson's disease, peptic ulcer disease, and psoriasis (Supplementary Table S2 online). To further evaluate the associations of continuous nephrology care with mortality in various types of comorbidities, we grouped these comorbidities into four main categories based on their pathophysiological similarity and potential for co-management [14-16].

\subsection{Death and Other Covariates}

Death after dialysis was determined by the discharge status of last admission hospitalization (death, against advising discharge and discharge under critical conditions), and there were no medical records one year after the last admission date. We also collected information on patient characteristics, namely: age, sex (male or female), insurance amount [fixed premium or dependent, $<20,000,20,000-39,999$, and $\geq 39,999$ New Taiwan dollars ( 1333 US dollars)], region (north, center, south, and east), urbanization of residence (urban or rural), Charlson comorbidity index score, and potential confounding drug prescriptions (anticoagulation, antiplatelet, or antidiabetic agents, insulin, steroid, and non-steroidal anti-inflammatory drugs). All these data were derived from outpatient claims by ATC codes (Supplementary Table S3 online). Charlson comorbidity index scores were calculated based on diseases and the formula reported in a previous study [17], while prescribing listing confounding medicines over 90 days within 3 years before dialysis was considered per user.

\subsection{Statistical Analysis}

We mainly displayed the group of $\geq 1$ year continuous nephrology care and its counterparts to reflect the associations of covariates with receiving continuous nephrology care. Continuous and categorical data were described by mean \pm standard deviation or median (interquartile range) and percentage, respectively. The differences of patient characteristics between groups were evaluated by independent $t$-tests for continuous variables and $\chi^{2}$ tests for categorical variables. PS approaches were adopted to estimate the average causal effect of nephrology care on mortality. The estimated PSs of different definitions of nephrology care were generated by multiple logistic and nominal regression models that were developed through forced entering selected covariates. One-to-one greedy, with exact sex and without replacement, a 0.2 caliper distance, the PS matching approach was applied for groups of receiving or not receiving nephrology care, and the balance of each covariate after matching by the standardized difference was assessed. The average cumulative nephrology effect was classified by the eight different groups in the population using different weights to balance all covariate distributions. We further inspected the relationships between the main factors, age and number of comorbidities, and the cumulative nephrology care by heat map. The one-year mortality proportions after dialysis were described by the Kaplan Meier approach, and the differences between groups assessed by the Gehan-Breslow-Wilcoxon test. Due to violating proportional hazard assumption, the interaction term of time and nephrology care was applied in the Cox proportional hazards regression model. The effect of nephrology care on mortality at one year after dialysis started was displayed by hazard 
ratio (HR) and 95\% confidence interval (CI). All statistical operations were performed using SAS (version 9.4, SAS Institute, Cary, NC, USA) and the mnps function in the twang package of R software (version 4.0, Taipei, Taiwan) [18].

\section{Results}

\subsection{Patient Characteristics}

To maintain study validity, we adopted the strict criteria to include our study subjects from databases and excluded all those who had ever received renal transplantation, were not adults, or lacked relevant information (Figure 1).

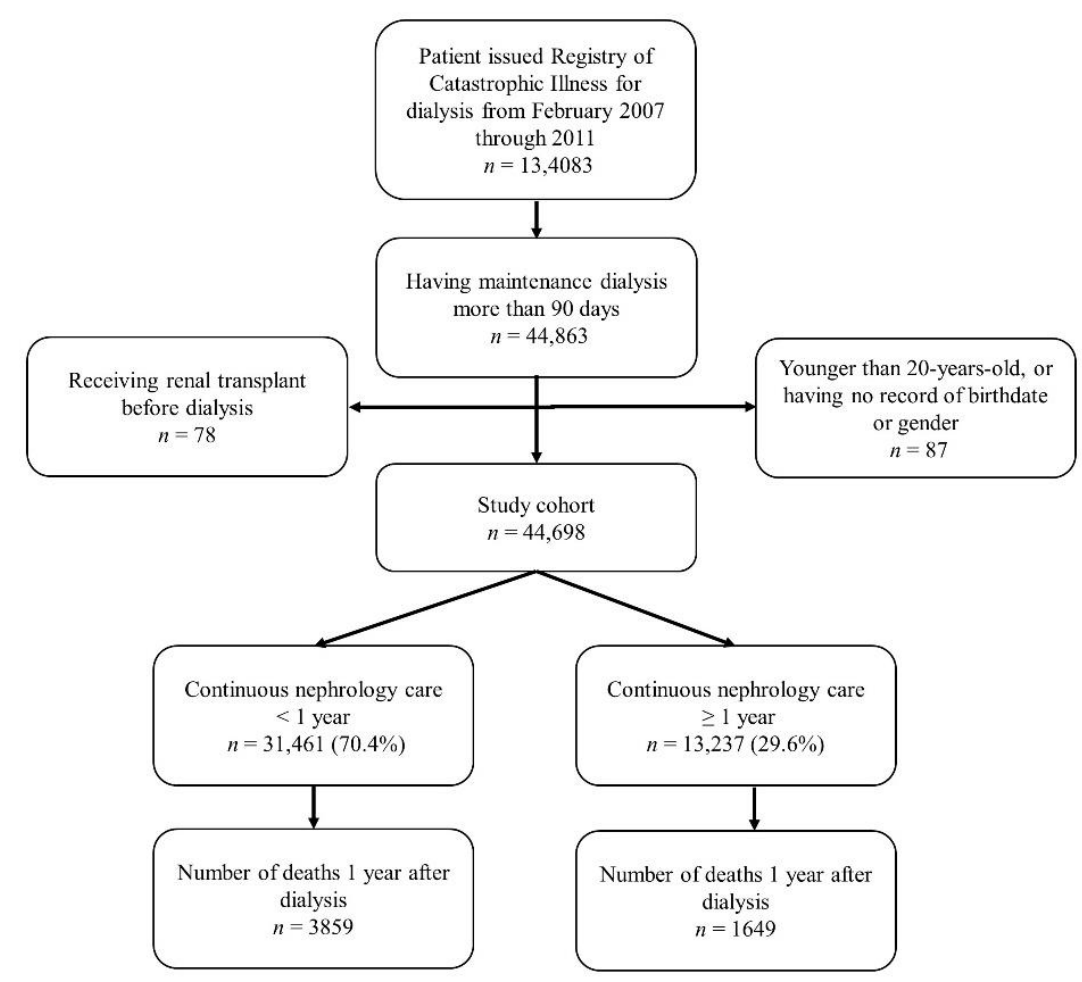

Figure 1. Study design flow chart.

Among the study patients ( $n=44,698$ ), only nearly $30 \%$ of patients with CKD received nephrology care for more than one year. Compared to patients without regular nephrology care, patients with nephrology care time $\geq 1$ year were significantly more likely to be of old age ( $64.8 \pm 13.3$ vs. $62.7 \pm 14.5$ years, $p<0.001)$, females ( 53.2 vs. $42.0 \%, p<0.001)$, living north, in an urban region, and with higher income, greater severity of diseases, more steroid prescriptions, but with fewer antidiabetic agents and NSAIDs prescriptions (Table 1). Compatible baseline characteristics between the groups with a range of standardized differences $(-0.05,0.01)$ were achieved after carrying out PS matching. 
Table 1. Patient characteristics.

\begin{tabular}{|c|c|c|c|c|c|c|c|}
\hline & \multirow{3}{*}{ Overall } & Before & atched & \multirow{3}{*}{$p$-Value } & After & tched & \multirow{3}{*}{$\begin{array}{c}\text { Standardized } \\
\text { Difference }\end{array}$} \\
\hline & & \multicolumn{2}{|c|}{$\begin{array}{c}\text { Continuous Nephrology Care } \geq \\
1 \text { Year }\end{array}$} & & \multicolumn{2}{|c|}{$\begin{array}{c}\text { Continuous Nephrology Care } \geq \\
1 \text { Year }\end{array}$} & \\
\hline & & No & Yes & & No & Yes & \\
\hline $\mathrm{N}$ & 44,698 & $31,461(70.4)$ & $13,237(29.6)$ & & 13,193 & 13,193 & \\
\hline Age, year & $63.3 \pm 14.2$ & $62.7 \pm 14.5$ & $64.8 \pm 13.3$ & $<0.001$ & $65.0 \pm 14.2$ & $64.9 \pm 13.4$ & -0.01 \\
\hline Female sex & $21,509(48.1)$ & $14,467(46.0)$ & $7042(53.2)$ & $<0.001$ & $7017(53.2)$ & $7017(53.2)$ & 0.0 \\
\hline \multicolumn{2}{|c|}{ Geographical region } & & & $<0.001$ & & & - \\
\hline North & $19,464(43.6)$ & $13,586(43.2)$ & $5878(44.4)$ & & $5927(44.9)$ & $5860(44.4)$ & \\
\hline Central & $9850(22.0)$ & $6978(22.2)$ & $2872(21.7)$ & & $2905(22.0)$ & $2862(21.7)$ & \\
\hline South & $13,943(31.2)$ & 9818 (31.2) & 4125 (31.2) & & $4003(30.3)$ & 4109 (30.2) & \\
\hline East & $1441(3.2)$ & $1079(3.4)$ & $362(2.7)$ & & $358(2.7)$ & $362(2.7)$ & \\
\hline \multicolumn{2}{|c|}{ Urbanization level } & & & $<0.001$ & & & $<0.001$ \\
\hline Rural & $12,642(28.3)$ & $9214(29.3)$ & $3428(25.9)$ & & $3394(25.7)$ & $3423(26.0)$ & \\
\hline Urban & $32,056(71.7)$ & $22,247(70.7)$ & $9809(74.1)$ & & $9799(74.3)$ & $9770(74.1)$ & \\
\hline \multicolumn{2}{|c|}{ Premium income, NTD } & & & $<0.001$ & & & - \\
\hline Dependent & $15,820(35.4)$ & $10,956(34.8)$ & $4864(36.7)$ & & $4832(36.6)$ & $4849(36.8)$ & \\
\hline$<20,000$ & $9837(22.0)$ & $7338(23.3)$ & $2499(18.9)$ & & $2434(18.5)$ & $2497(18.9)$ & \\
\hline $20,000-39,999$ & $16,556(37.0)$ & $11,607(36.9)$ & $4949(37.4)$ & & 5049 (38.3) & $4,937(37.4)$ & \\
\hline$\geq 40,000$ & $2485(5.6)$ & $1560(5.0)$ & $925(7.0)$ & & $878(6.7)$ & $910(6.9)$ & \\
\hline \multicolumn{2}{|c|}{ Charlson index } & & & $<0.001$ & & & - \\
\hline 0 & 3168 (7.1) & $2852(9.1)$ & $316(2.4)$ & & $320(2.4)$ & $316(2.4)$ & \\
\hline $1-2$ & $15,061(33.7)$ & $9995(31.8)$ & $5066(38.3)$ & & $5098(38.6)$ & $5051(38.3)$ & \\
\hline $3-4$ & $16,149(36.1)$ & $11,209(35.6)$ & $4940(37.3)$ & & $4824(36.6)$ & $4921(37.3)$ & \\
\hline $5-6$ & $8737(19.6)$ & $6250(19.9)$ & $2487(18.8)$ & & $2510(19.0)$ & $2478(18.8)$ & \\
\hline$\geq 7$ & $1583(3.5)$ & $1155(3.7)$ & $428(3.2)$ & & $441(3.3)$ & $427(3.2)$ & \\
\hline \multicolumn{8}{|c|}{ Confounding drugs } \\
\hline Anticoagulation agents & $626(1.4)$ & $450(1.4)$ & $176(1.3)$ & 0.41 & $165(1.25)$ & 175 (1.33) & -0.01 \\
\hline Antiplatelet agents & $3893(8.7)$ & $2767(8.8)$ & $1126(8.5)$ & 0.32 & $1055(8.0)$ & $1117(8.5)$ & -0.02 \\
\hline Antidiabetic agents & $16,770(37.5)$ & $12,721(40.4)$ & 4049 (30.6) & $<0.001$ & $4102(31.1)$ & $4048(30.7)$ & 0.01 \\
\hline Insulin & $7028(15.7)$ & 5015 (15.9) & $2013(15.2)$ & 0.05 & $1985(15.1)$ & 2010 (15.2) & -0.01 \\
\hline Steroid & $2730(6.1)$ & $1531(4.9)$ & $1199(9.1)$ & $<0.001$ & 995 (7.5) & $1161(8.8)$ & -0.05 \\
\hline NSAIDs & $3846(8.6)$ & $2915(9.3)$ & $931(7.0)$ & $<0.001$ & $930(7.1)$ & $931(7.1)$ & $<0.001$ \\
\hline
\end{tabular}

Abbreviation: NTD, New Taiwan dollar; NSAIDs, non-steroidal anti-inflammatory drugs; PS, propensity score. Data represented as mean \pm s.d. for continuous variables and count (percentage) for categorical variables. Different between-group distributions were analyzed using the independent $t$ test, and $\chi^{2}$ test. $p$ value less than 0.05 was considered significant. The propensity score was estimated by a multiple logistic regression model by including all covariates listed in Tables 1 and 2 as predictors. We performed propensity score one-to-one matching through the Greedy approach and evaluated the balance of covariates between the two nephrology care groups.

Table 2. Prevalence of comorbidity by the duration of nephrology care.

\begin{tabular}{|c|c|c|c|c|c|c|c|}
\hline & \multirow{3}{*}{ Overall } & Before & atched & \multirow{3}{*}{$p$-Value } & After & ched & \multirow{3}{*}{$\begin{array}{l}\text { Standardized } \\
\text { Difference }\end{array}$} \\
\hline & & \multicolumn{2}{|c|}{$\begin{array}{c}\text { Continuous Nephrology Care } \geq \\
1 \text { Year }\end{array}$} & & \multicolumn{2}{|c|}{$\begin{array}{c}\text { Continuous Nephrology Care } \geq \\
1 \text { Year }\end{array}$} & \\
\hline & & No & Yes & & No & Yes & \\
\hline $\mathrm{N}$ & 44,698 & $31,461(70.4)$ & $13,237(29.6)$ & & 13,193 & 13,193 & \\
\hline \multicolumn{8}{|c|}{ Concordant comorbidities ${ }^{a}$} \\
\hline Atrial fibrillation & $238(0.5)$ & $159(0.5)$ & $79(0.6)$ & 0.23 & $72(0.5)$ & $79(0.6)$ & -0.01 \\
\hline Chronic heart failure & $6444(14.4)$ & 5177 (16.5) & $1267(9.6)$ & $<0.001$ & $1283(9.7)$ & $1267(9.6)$ & $<0.001$ \\
\hline Diabetes & $21,668(48.5)$ & $16,166(51.4)$ & $5502(41.6)$ & $<0.001$ & $5546(42.0)$ & $5498(41.7)$ & 0.01 \\
\hline Hypertension & $23,643(52.9)$ & $16,971(53.9)$ & $6672(50.4)$ & $<0.001$ & $6778(51.4)$ & $6655(50.4)$ & 0.02 \\
\hline $\begin{array}{c}\text { Peripheral vascular } \\
\text { disease }\end{array}$ & $100(0.2)$ & $75(0.2)$ & $25(0.2)$ & 0.31 & $24(0.2)$ & $25(0.2)$ & $<0.001$ \\
\hline Stroke or TIA & 4857 (10.9) & $3780(12.0)$ & $1,077(8.1)$ & $<0.001$ & $1102(8.4)$ & $1077(8.2)$ & 0.01 \\
\hline \multicolumn{8}{|c|}{ Discordant comorbidities ${ }^{a}$} \\
\hline Asthma & $779(1.7)$ & $582(1.9)$ & $197(1.5)$ & 0.007 & $184(1.4)$ & $197(1.5)$ & -0.01 \\
\hline Cancer, lymphoma & $97(0.2)$ & $70(0.2)$ & $27(0.2)$ & 0.7 & $22(0.2)$ & $27(0.2)$ & -0.01 \\
\hline Cancer, metastatic & $79(0.2)$ & $57(0.2)$ & $22(0.2)$ & 0.73 & $19(0.1)$ & $22(0.2)$ & -0.01 \\
\hline Cancer, non-metastatic & $1108(2.5)$ & $713(2.3)$ & $395(3.0)$ & $<0.001$ & $376(2.9)$ & $392(3.0)$ & -0.01 \\
\hline $\begin{array}{c}\text { Chronic pulmonary } \\
\text { disease }\end{array}$ & $2401(5.4)$ & $1727(5.5)$ & $674(5.1)$ & 0.09 & $611(4.6)$ & $670(5.1)$ & -0.02 \\
\hline Constipation, severe & $2062(4.6)$ & $1441(4.6)$ & $621(4.7)$ & 0.6 & $604(4.6)$ & $620(4.7)$ & -0.01 \\
\hline Dementia & $890(2.0)$ & $624(2.0)$ & $266(2.0)$ & 0.86 & $267(2.0)$ & $266(2.0)$ & $<0.001$ \\
\hline $\begin{array}{c}\text { Inflammatory bowel } \\
\text { disease }\end{array}$ & $245(0.6)$ & $177(0.6)$ & $68(0.5)$ & 0.52 & $60(0.5)$ & $68(0.5)$ & -0.01 \\
\hline Rheumatoid arthritis & $828(1.9)$ & $582(1.9)$ & $246(1.9)$ & 0.95 & $233(1.8)$ & $246(1.9)$ & -0.01 \\
\hline \multicolumn{8}{|c|}{ Mental disease and chronic pain a } \\
\hline Alcohol misuse & $169(0.4)$ & $155(0.5)$ & $14(0.1)$ & $<0.001$ & $14(0.1)$ & $14(0.1)$ & $<0.001$ \\
\hline Chronic pain & $11,711(26.2)$ & $8161(25.9)$ & $3550(26.8)$ & 0.05 & $3487(26.4)$ & $3536(26.8)$ & -0.01 \\
\hline Depression & $923(2.1)$ & $587(1.9)$ & $336(2.5)$ & $<0.001$ & $305(2.3)$ & $329(2.5)$ & -0.01 \\
\hline Schizophrenia & $175(0.4)$ & $137(0.4)$ & $38(0.3)$ & 0.02 & $32(0.2)$ & $38(0.3)$ & -0.01 \\
\hline \multicolumn{8}{|l|}{ Other comorbidities a } \\
\hline Chronic hepatitis B & $513(1.2)$ & $339(1.1)$ & $174(1.3)$ & 0.03 & $162(1.2)$ & $170(1.3)$ & -0.01 \\
\hline
\end{tabular}


Table 2. Cont.

\begin{tabular}{|c|c|c|c|c|c|c|c|}
\hline & \multirow{3}{*}{ Overall } & \multirow{2}{*}{\multicolumn{2}{|c|}{$\begin{array}{c}\text { Before PS Matched } \\
\text { Continuous Nephrology Care } \geq \\
1 \text { Year }\end{array}$}} & \multirow{3}{*}{$p$-Value } & Aft & hed & \multirow{3}{*}{$\begin{array}{l}\text { Standardized } \\
\text { Difference }\end{array}$} \\
\hline & & & & & \multicolumn{2}{|c|}{$\begin{array}{c}\text { Continuous Nephrology Care } \geq \\
1 \text { Year }\end{array}$} & \\
\hline & & No & Yes & & No & Yes & \\
\hline Cirrhosis & $924(2.1)$ & $698(2.2)$ & $226(1.7)$ & $<0.001$ & $211(1.6)$ & $225(1.7)$ & -0.01 \\
\hline Epilepsy & $187(0.4)$ & $130(0.4)$ & $57(0.4)$ & 0.8 & $56(0.4)$ & $57(0.4)$ & $<0.001$ \\
\hline \multirow{2}{*}{$\begin{array}{l}\text { Hypothyroidism } \\
\text { Irritable bowel } \\
\text { syndrome }\end{array}$} & $210(0.5)$ & $130(0.4)$ & $80(0.6)$ & 0.007 & $67(0.5)$ & $78(0.6)$ & -0.01 \\
\hline & $510(1.1)$ & 332 (1.1) & 178 (1.3) & 0.009 & $173(1.3)$ & $176(1.3)$ & $<0.001$ \\
\hline Multiple sclerosis & $62(0.1)$ & $45(0.1)$ & $17(0.1)$ & 0.70 & $18(0.1)$ & $17(0.1)$ & $<0.001$ \\
\hline Myocardial infarction & $1045(2.3)$ & $861(2.7)$ & $184(1.4)$ & $<0.001$ & $186(1.4)$ & $184(1.4)$ & $<0.001$ \\
\hline Parkinson's disease & $531(1.2)$ & $357(1.13)$ & $174(1.3)$ & 0.11 & $172(1.3)$ & $174(1.3)$ & $<0.001$ \\
\hline Peptic ulcer disease & $3103(6.9)$ & $2072(6.6)$ & $1031(7.8)$ & $<0.001$ & 967 (7.3) & $1023(7.8)$ & -0.02 \\
\hline Psoriasis & $258(0.6)$ & $168(0.5)$ & $90(0.7)$ & 0.06 & $79(0.6)$ & $87(0.7)$ & -0.01 \\
\hline
\end{tabular}

Abbreviations: TIA, transient ischemic attack. ${ }^{a}$ Comorbidities were classified as concordant, discordant, mental disease and chronic pain, and others based on their pathophysiological similarity and potential for co-management. Data represented as mean \pm s.d. for continuous variables and count (percentage) for categorical variables. Different between-group distributions were analyzed using the independent $t$ test, and $\chi^{2}$ test. $p$ value lower than 0.05 was considered significant. Propensity score was estimated by multiple logistic regression model by including all covariates listed in Tables 1 and 2 as predictors. We performed propensity score one-to-one matching through the Greedy approach and evaluated the balance of covariates between the two nephrology care groups.

\subsection{Comorbidity and Nephrology Care}

CKD in patients is mainly accompanied by hypertension (52.9\%), diabetes $(48.5 \%)$, and chronic pain $(26.2 \%)$ (Table 2). A significantly higher prevalence of chronic heart failure $(16.5 \%$ vs. $9.6 \%, p<0.001)$, diabetes $(51.4 \%$ vs. $41.6 \%, p<0.001)$, hypertension $(53.9 \%$ vs. $50.4 \%, p<0.001)$, stroke $(12.0 \%$ vs. $8.1 \%, p<0.001)$, asthma $(1.9 \%$ vs. $1.5 \%$, $p<0.001)$, alcohol misuse $(0.5 \%$ vs. $0.1 \%, p<0.001)$, cirrhosis $(2.2 \%$ vs. $1.7 \%, p<0.001)$, and myocardial infarction $(2.7 \%$ vs. $1.4 \%, p<0.001)$ were observed in the group of noncontinuous nephrology care. However, patients who received nephrology care for more than one year present a higher prevalence of non-metastatic cancer $(3.0 \%$ vs. $2.3 \%, p<0.001)$, depression $(2.5 \%$ vs. $1.9 \%, p<0.001)$, chronic hepatitis B $(1.3 \%$ vs. $1.1 \%, p<0.05)$, irritable bowel syndrome $(1.3 \%$ vs. $1.1 \%, p<0.05)$ and peptic ulcer disease $(7.8 \%$ vs. $6.6 \%, p<0.001)$. After PS matching, similar distributions in listed comorbidities between the groups were obtained.

\subsection{Distribution and Nephrology Care}

Nearly half of the study participants did not receive regular nephrology care in the 3-year observational period before dialysis, or only had regular nephrology care until 6 months before dialysis. It is worth noting that a few patients (7.5\%) intermittently received nephrology care (Figure 2a). The relationships between age groups, the number of comorbidities, and cumulative nephrology care are presented in the heat map. Young patients (age 20-44 years) showed a greater probability of being in the group of limited nephrology care in the 3-year observational period before dialysis (Figure 2b). Patients who never received or received regular nephrology care $\geq 30$ months have the highest proportion of one comorbidity; whereas those who intermittently received or received regular nephrology care $0-6,0-12$, or $0-18$ months have the highest proportion of two comorbidities (Figure 2c). 


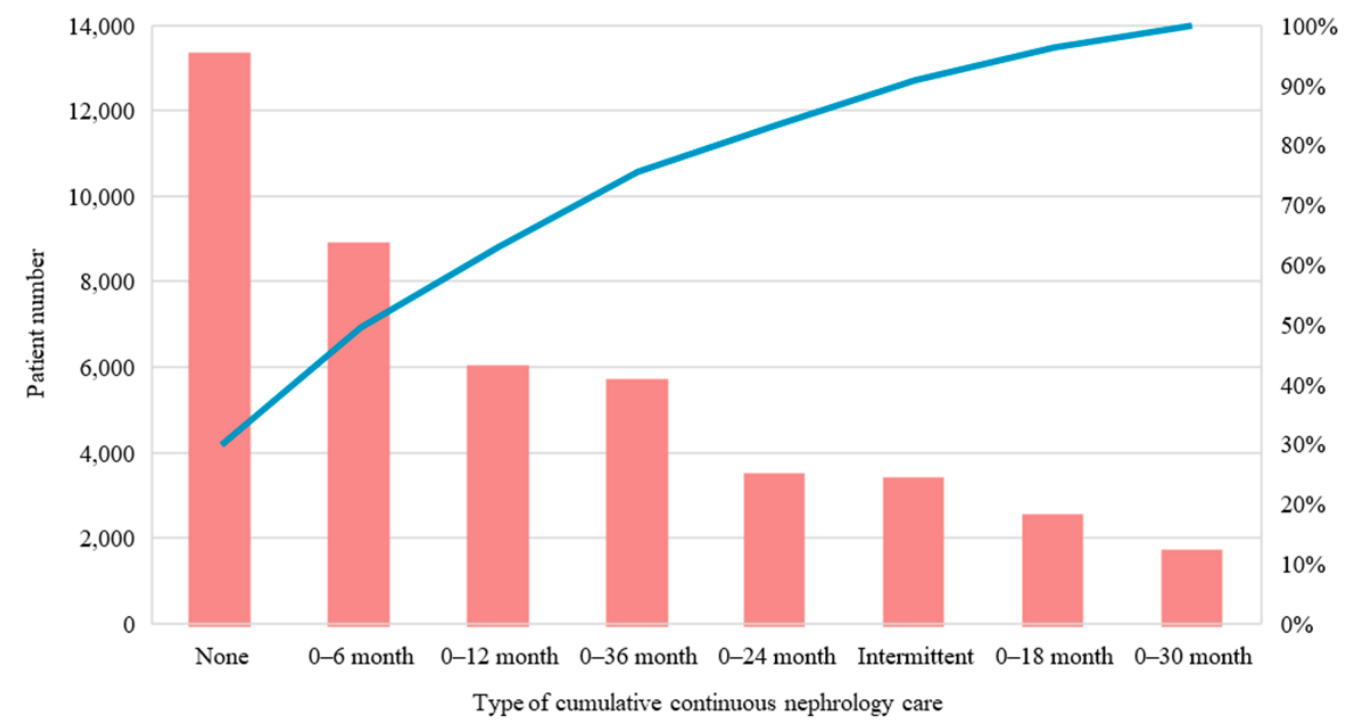

(a)

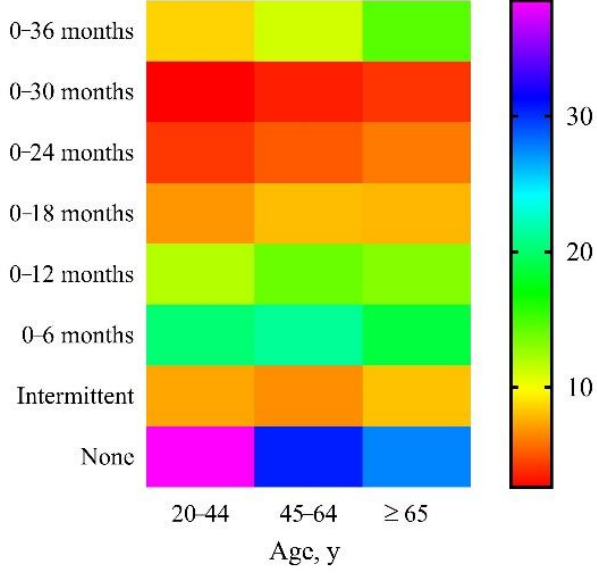

(b)

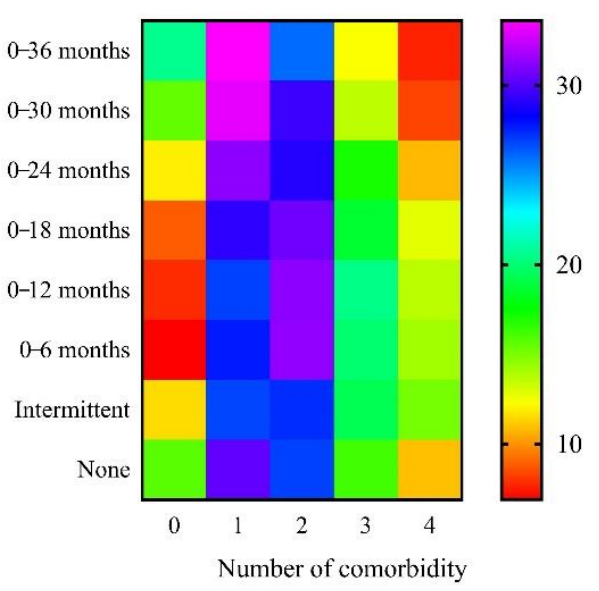

(c)

Figure 2. Distribution in nephrology care. (a) Patient number in different types of nephrology care and their cumulative percentage; (b) Heat map comparing proportions of types of cumulative nephrology care between age groups (20-44, 45-64, and $\geq 65$ years); (c) Heat map comparing proportions of types of cumulative nephrology care in patients with different comorbidity numbers $(0,1,2,3$, and $\geq 4)$.

\subsection{Nephrology Care and Mortality}

The cumulative mortality proportion in the group of continuous nephrology care $\geq$ 1 year was lower than those in the counter group $(8.2 \%$ vs. $12.3 \% ; p<0.001$ by GehanBreslow-Wilcoxon test) (Figure 3a). After dividing by eight exclusive groups, the cumulative mortality proportion was highest in the groups of no care and intermittently receiving nephrology care (14.4 and 14.5\%), compared to those in the other groups (Figure 3b). 


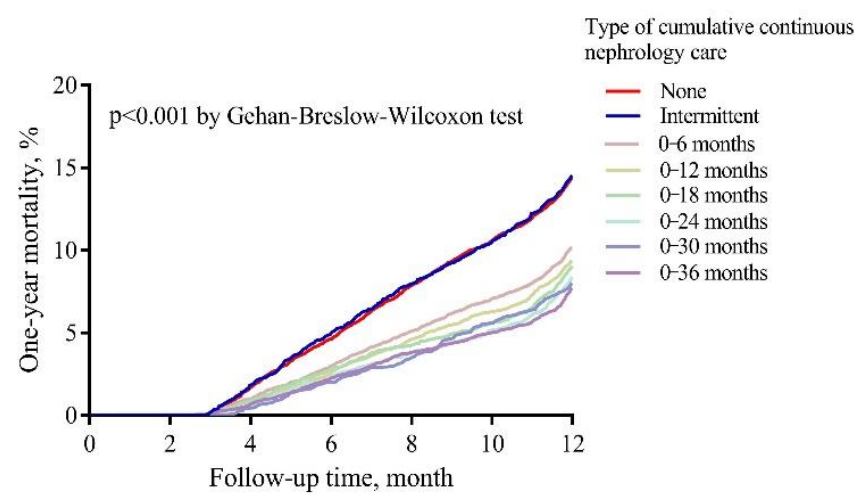

(a)

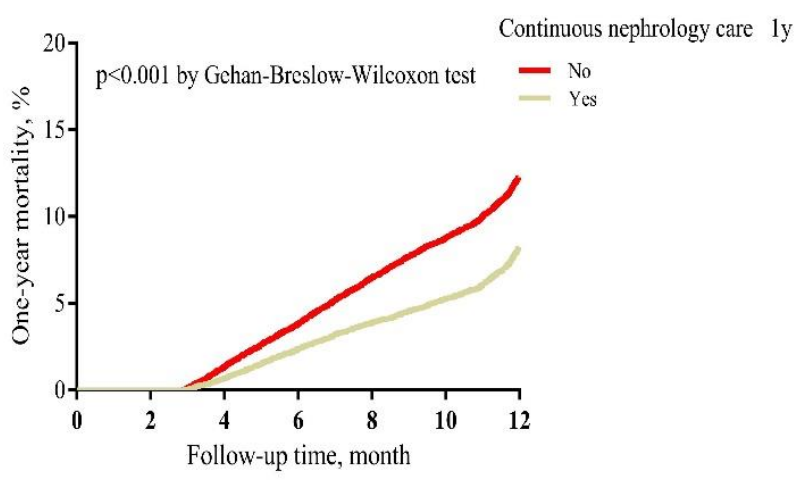

(b)

Figure 3. Cumulative mortality proportion between (a) $\geq 1$ year vs. $<1$ year nephrology care; (b) types of cumulative nephrology care. The time was calculated from the date of dialysis initiation to the date of death, or the end date of the first year, whichever came first. Cumulative one-year mortality proportions were estimated by the Kaplan Meier approach and assessed the differences between groups by Gehan-Breslow-Wilcoxon test.p $<0.001$.

The time-dependent HRs are in Figure 4. The survival benefit for the group of continuous nephrology care $\geq 1$ year before dialysis initiation gradually decreased over time, and an average lower 22\% mortality hazard (PS-matching HR: 0.78, 95\% CI: 0.74-0.82) compared with those in the group of continuous nephrology care $<1$ year was demonstrated at the end of one year after the start of dialysis (Figure 4).

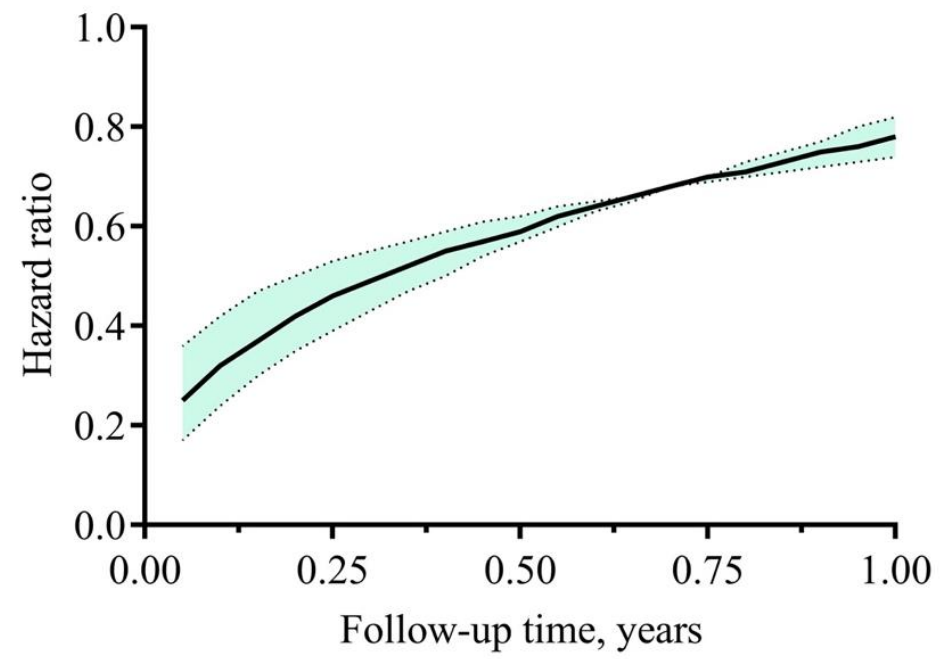

Figure 4. The time-dependent hazard ratio of one-year mortality comparing patients receiving nephrology care $\geq 1$ year with those $<1$ year. The estimated PSs of receiving nephrology care were generated by multiple logistic regression models that were developed through forced entering selected covariates. One-to-one Greedy, with exact sex and without replacement, a 0.2 caliper distance, the PS matching approach was applied for groups of receiving, or not receiving nephrology care. Cox proportional hazards regression model with added interaction term of time and nephrology care was applied in the matched cohort.

In addition, we observed that the continuous average nephrology care effect on mortality (ranges of PS-matching HR: 0.77-0.80) when we applied different periods (0-6, $0-12, \ldots, 0-36)$ to determine continuous nephrology care and made matching approaches by PS scores (Table 3 ). 
Table 3. Effects of consistent nephrology care at each time interval on one-year mortality after dialysis.

\begin{tabular}{|c|c|c|c|c|c|c|}
\hline $\begin{array}{l}\text { Time Window } \\
\text { before Dialysis, } \\
\text { Month }\end{array}$ & $\begin{array}{l}\text { Consistent } \\
\text { Nephrology Care }\end{array}$ & Case, $n$ & $\begin{array}{c}\text { Number of Death, } \\
n\end{array}$ & $\begin{array}{l}\text { Follow-Up Time, } \\
\text { Person-Years }\end{array}$ & $\begin{array}{c}\text { Mortality Rate }(95 \% \mathrm{CI}), \\
\text { per } 1000 \text { Patient-Years }\end{array}$ & $\begin{array}{l}\text { PS-Matching HR } \\
(95 \% \mathrm{CI})\end{array}$ \\
\hline \multirow[b]{2}{*}{$0-6$} & No & 16,636 & 2401 & 15,765 & $152.3(146.3-158.5)$ & 1.00 [reference] \\
\hline & Yes & 28,062 & 2546 & 27,228 & $93.5(89.9-97.2)$ & $0.79(0.75-0.83)$ \\
\hline \multirow{2}{*}{$0-12$} & No & 25,478 & 3298 & 24,303 & $135.7(131.2-140.4)$ & 1.00 [reference] \\
\hline & Yes & 19,220 & 1649 & 18,691 & $88.2(84.1-92.6)$ & $0.78(0.74-0.82)$ \\
\hline \multirow{2}{*}{$0-18$} & No & 31,461 & 3859 & 30,102 & $128.2(124.2-132.3)$ & 1.00 [reference] \\
\hline & Yes & 13,237 & 1088 & 12,892 & $84.4(79.5-89.6)$ & $0.78(0.74-0.82)$ \\
\hline \multirow{2}{*}{$0-24$} & No & 34,918 & 4172 & 33,458 & $124.7(121.0-128.5)$ & 1.00 [reference] \\
\hline & Yes & 9780 & 775 & 9535 & $81.3(75.8-87.2)$ & $0.78(0.73-0.83)$ \\
\hline \multirow{2}{*}{$0-30$} & No & 37,390 & 4379 & 35,867 & $122.1(118.5-125.8)$ & 1.00 [reference] \\
\hline & Yes & 7308 & 568 & 7126 & $79.7(73.4-86.5)$ & $0.80(0.74-0.86)$ \\
\hline \multirow{2}{*}{$0-36$} & No & 39,050 & 4512 & 37,485 & $120.4(116.9-123.9)$ & 1.00 [reference] \\
\hline & Yes & 5648 & 435 & 5508 & $79.0(71.9-86.8)$ & $0.77(071-0.84)$ \\
\hline
\end{tabular}

Compared to patients without nephrology care, a stepped reduction in HR was identified when patients received more nephrology care. Patients who received continuous nephrology care over two years presented the lowest HRs (ranges of weighted HR: 0.480.52 ), followed by patients who received continuous nephrology care from six months to two years (ranges of weighted HR: 0.60-0.65), and patients with intermittent nephrology care (weighted HR: 0.88, 95\% CI: 0.79-0.97) (Table 4).

Table 4. Effects of cumulative continuous nephrology care on one-year mortality after dialysis.

\begin{tabular}{|c|c|c|c|c|c|}
\hline $\begin{array}{l}\text { Type of Continuous } \\
\text { Nephrology Care }\end{array}$ & Case, $n$ & $\begin{array}{c}\text { Number of Deaths, } \\
n\end{array}$ & $\begin{array}{l}\text { Follow-Up Time, } \\
\text { Person-Year }\end{array}$ & $\begin{array}{c}\text { Mortality Rate }(95 \% \text { CI), } \\
\text { per } 1000 \text { Patient-Years }\end{array}$ & $\begin{array}{l}\text { Weighted HR } \\
(95 \% \text { CI) }\end{array}$ \\
\hline None & 13,297 & 1916 & 12,595 & $152.1(145.5-159.1)$ & 1.00 [reference] \\
\hline Intermittent & 3339 & 485 & 3160 & 153.5 (140.4-167.8) & $0.88(0.79-0.97)$ \\
\hline 0-6 month & 8842 & 897 & 8531 & $105.1(98.5-112.3)$ & $0.65(0.60-0.71)$ \\
\hline 0-12 month & 5983 & 561 & 5795 & $96.8(89.1-105.2)$ & $0.60(0.54-0.66)$ \\
\hline 0-18 month & 3457 & 313 & 3354 & 93.3 (83.5-104.3) & $0.60(0.52-0.69)$ \\
\hline 0-24 month & 2472 & 207 & 2407 & $86.0(75.1-98.6)$ & $0.48(0.41-0.57)$ \\
\hline 0-30 month & 1660 & 133 & 1617 & 82.3 (69.4-97.5) & $0.50(0.41-0.60)$ \\
\hline 0-36 month & 5648 & 435 & 5504 & $79.0(71.9-86.8)$ & $0.52(0.46-0.59)$ \\
\hline
\end{tabular}

Abbreviation: $\mathrm{CI}$, confidence interval; $\mathrm{HR}$, hazard ratio. The mortality rate was calculated by the number of deaths divided by the follow-up time and its $95 \% \mathrm{CI}$ was estimated by Poisson regression model. The propensity score for continuous nephrology care within 0-36 month time-window was estimated by a multiple nominal regression model that was developed through forced entering variables including age, sex, urbanization level, premium income, Charlson index, type of presenting selected comorbidity (concordant, disconcordant, mental, and others), and confounding drugs (anticoagulation agents, antiplatelet agents, antidiabetic agents, insulin, steroid, and non-steroidal anti-inflammatory drugs). Inverse probability weighted for each patient was estimated by the twang package of R software [18].

\section{Discussion}

The study systematically identified that patients younger than 45 years or having two comorbidities were associated with limited nephrology care in a 3-year observational period before dialysis. The benefit of nephrology care for mortality was large during dialysis initiation and was followed by a gradual shrinkage trend to an average $22 \%$ lower at one year after dialysis. There were no significant differences in the benefit of nephrology care for mortality when we defined continuous nephrology care by different periods. A stepped dose-response effect of nephrology care on mortality was observed, with patients receiving continuous nephrology care for over two years, care for six months to two years, intermittent nephrology care, and no nephrology care, responding progressively worse.

Although timely receipt of nephrology care in CKD was generally suggested by recent nephrology guidelines [10], there is still room to improve the content of nephrology care that influences the mortality in the dialysis population. Liu et al. observed a large patient cohort with CKD stage 4 and found that, once patients had outpatient nephrology visits, this was associated with a $12 \%$ lower mortality risk [19], but a slight increase in dialysis probability [20]. Another earlier study finds that late care ( $<6$ months before dialysis), lower cumulative care ( $\leq 5$ nephrology visits within a 3-year period), and inconsistent critical period care ( $\geq 3$ of the 6 months before dialysis) are independent factors associated with mortality within one-year after dialysis begins [20]. Yang et al. further combined 
the concept of early nephrology referral determined by $\geq 6$ months before dialysis and frequency of nephrology visit to demonstrate that a $\sim 10 \%$ lower 1-year post-dialysis major adverse cardiovascular events could be observed in patients with early-frequent nephrology care, compared to those with late nephrology-care [6]. Unlike the definitions used in these studies, our approach of determining nephrology care every 6 months until 3 years before dialysis is more objective than others. Furthermore, estimating the average care effects using causal inference methods could also provide new insights in encouraging nephrology care to be implemented as early as 2 years before dialysis, not just at any arbitrary time period.

The optimal frequency of nephrology care has not been suggested previously. We identified that, once patients received intermittent nephrology care, this could bring some benefits upon mortality risk. However, extra survival benefits could occur if they extended nephrology care further. A recent study also supported these findings, emphasizing the need for nephrology care length and consistency for improving major adverse events in post-dialysis periods [6]. The mechanisms under these findings are unknown and should involve characteristics of the patient, disease, care, and their interactions. It is reasonable to speculate that patients with more cumulative nephrology care should get extra benefits from nephrology care through the early detection and treatment of complications, as well as the strict control of ill conditions. In addition, patients may modify their behavior to reflect disease requirement suggestions by a nephrologist. More research on exploring the difference in patient-physician behaviors during pre-ESKD/CKD phases is warranted.

PS-based methods have been widely applied in observational studies to determine the effect size of intervention on outcomes conditions on the similar distributions of covariates between intervention groups. Both PS matching and weighted methods are popularly used to estimate average intervention effects. In our study, we used the PS matching method for two care group comparisons and the weighted approach for unbalanced and multiple care group comparisons. It is worth noting the main conceptual difference between these two methods. The matching approach reflects the average intervention effect only among those who ultimately received intervention, while the weighted one estimates the average intervention effect in the scenario in which every patient within the population was offered intervention [21]. Combined with suggestions from the Kidney Disease: Improving Global Outcomes guidelines [22] and recent findings [23], our findings advocate that patients with CKD should continuously receive nephrology care for at least two years.

Age and comorbidity in CKD were the main factors associated with continuous receipt of nephrology care [24]. Younger CKD patients may be preoccupied with their jobs, causing them to seek nephrology care less frequently. On the other hand, patients who may have more comorbidities may face similar situations, since a physician caring for their primary conditions, (such as hypertension or diabetes mellitus), may be too late to detect CKD until it has progressed to end-stage [4]. The above findings suggest that different care models for these patients should be developed to promote prompt nephrology referral and adherence to nephrology care.

Several advantages of our study are worth pointing out. The single and less restrictive healthcare reimbursement system ensures that most decisions for receiving nephrology care were determined by a physician's judgment rather than reimbursement policy. Thus, nephrology care in this study was defined by a more practical approach, which enables easier translation of the results into clinical practice. Finally, we adopted more complete methods for defining comorbidity and making a causal inference, validating our findings in a manner similar to results from randomized clinical trials.

Some limitations need to be declared. First, we could not rule out the influences of patient compliance on our inference. It is undeniable that compliant patients may manage their chronic diseases well and lead to better survival. Therefore, interpreting our results should be cautious. Second, the lack of patient behavior and laboratory information prevents us from exploring the mechanisms of nephrology care on mortality. In addition, we could not understand the impacts of medication compliance on our estimation from 
claim data. However, we believe the influences should be small because physicians had the ability to modify prescriptions at each medical visit when required. Fourth, the study was unable to determine the cause of renal disease. Although they only account for a small proportion of our study subjects, some etiologies can cause rapid renal function progression, resulting in the patients not having the same chance of having nephrology visits as those with slow renal progression. Finally, our patients were from the Taiwanese population covered by the NHI program and derived from older sample data, limiting their generalizability.

\section{Conclusions}

In conclusion, this study demonstrates that there was no critical period whereupon nephrology care produced a bigger effect on post-dialysis mortality, but stepped extra survival benefits were observed when extending nephrology care, which suggests that continuous and long-term nephrology care during the pre-ESKD/CKD phase is required.

Supplementary Materials: The following are available online at https://www.mdpi.com/article/ 10.3390/jpm11111071/s1, Table S1: The reimbursement codes for clinical treatment provided by the Taiwan National Health Insurance, Table S2: Comorbidity and corresponding ICD-9-CM codes, Table S3: Anatomical Therapeutic Chemical codes of drugs used concomitantly by patients during the study period.

Author Contributions: Conceptualization, P.-H.W., Y.-W.C. and M.-Y.L.; methodology, P.-H.W., Y.-W.C., C.-Y.C. and M.-Y.L.; software, M.-Y.L.; validation, P.-H.W., Y.-W.C. and M.-Y.L.; formal analysis, M.-Y.L.; investigation, M.-Y.L.; writing—original draft preparation, C.-Y.C. and M.-Y.L.; writing-review and editing, P.-H.W., Y.-W.C. and S.-J.H.; supervision, Y.-W.C. and S.-J.H.; project administration, Y.-W.C. and M.-Y.L.; funding acquisition, Y.-W.C., S.-J.H. and M.-Y.L. All authors have read and agreed to the published version of the manuscript.

Funding: This research was funded by the Ministry of Science and Technology (MOST: 105-2314B-037-065-, 106-2314-B-002-253- and 108-2314-B-037-110-), Kaohsiung Medical University Hospital (KMUH: 105-5R17 and 106-6R21), Center for Big Data Research to MYL, National Health Research Institutes (NHRI-EX106-10505PI, NHRI-EX108-10505PI and NHRI-EX110-10717PI), Kaohsiung Medical University Hospital (KMUH105-5R16) to SJH, and Ministry of Science and Technology (MOST: 1032314-B-037-033- and 104-2314-B-037-054), Kaohsiung Medical University Hospital (KMUH:103-3R10 and 104-4R11) to YWC.

Institutional Review Board Statement: This research was approved by the Institutional Review Board, Kaohsiung Medical University Hospital (KMUHIRB-E(I)-20160069) with exemption from the requirement for informed consent. All research procedures follow the guidelines of the Declaration of Helsinki.

Informed Consent Statement: The ethical review boards waived patient consent because all data used in the study have been de-identified.

Data Availability Statement: The datasets presented in this article are not readily available because these data used in our study should be acquired through formal application to the Health and Welfare Data Science Center, Department of Statistics, Ministry of Health and Welfare, Taiwan. Requests to access the datasets should be directed to https://dep.mohw.gov.tw/DOS/np-2497-113.html. Accessed on 21 September 2021.

Acknowledgments: We thank Feng Xuan Jian for preparing data for analysis and the Center for Medical Informatics and Statistics of Kaohsiung Medical University for providing administrative support.

Conflicts of Interest: The corresponding author serves as an editor of the special issue, Precision Prevention and Care in Chronic Kidney Disease in this journal. This does not alter the authors' adherence to JPM editorial policies and criteria. 


\section{References}

1. Jager, K.J.; Kovesdy, C.; Langham, R.; Rosenberg, M.; Jha, V.; Zoccali, C. A single number for advocacy and communicationworldwide more than 850 million individuals have kidney diseases. Nephrol. Dial. Transpl. 2019, 34, 1803-1805. [CrossRef]

2. Ku, E.; Lee, B.J.; Wei, J.; Weir, M.R. Hypertension in CKD: Core Curriculum 2019. Am. J. Kidney Dis. 2019, 74, 120-131. [CrossRef]

3. de Boer, I.H.; Rue, T.C.; Hall, Y.N.; Heagerty, P.J.; Weiss, N.S.; Himmelfarb, J. Temporal trends in the prevalence of diabetic kidney disease in the United States. JAMA 2011, 305, 2532-2539. [CrossRef]

4. $\quad$ Lin, M.-Y.; Lee, C.T.-C.; Kuo, M.-C.; Hwang, S.-J.; Chen, H.-C.; Chiu, Y.-W. Effects of physician's specialty on regular chronic kidney disease care in predialysis: A population-based cross-sectional study. Medicine 2018, 97, e11317. [CrossRef]

5. Webster, A.C.; Nagler, E.V.; Morton, R.L.; Masson, P. Chronic kidney disease. Lancet 2017, 389, $1238-1252$.

6. Yang, J.-Y.; Huang, J.-W.; Chen, L.; Chen, Y.-Y.; Pai, M.-F.; Tung, K.-T.; Peng, Y.-S.; Hung, K.-Y. Frequency of early predialysis nephrology care and postdialysis cardiovascular events. Am. J. Kidney Dis. 2017, 70, 164-172.

7. Singhal, R.; Hux, J.E.; Alibhai, S.M.; Oliver, M.J. Inadequate predialysis care and mortality after initiation of renal replacement therapy. Kidney Int. 2014, 86, 399-406.

8. Roubicek, C.; Brunet, P.; Huiart, L.; Thirion, X.; Leonetti, F.; Dussol, B.; Jaber, K.; Andrieu, D.; Ramananarivo, P.; Berland, Y. Timing of nephrology referral: Influence on mortality and morbidity. Am. J. Kidney Dis. 2000, 36, 35-41. [PubMed]

9. Rognant, N.; Laville, M. Early mortality in dialysis and adequacy of predialysis renal care: The picture is more complex than we thought. Kidney Int. 2014, 86, 238-240. [PubMed]

10. Levin, A.; Stevens, P.E. Summary of KDIGO 2012 CKD Guideline: Behind the scenes, need for guidance, and a framework for moving forward. Kidney Int. 2014, 85, 49-61. [CrossRef] [PubMed]

11. Hsieh, C.-Y.; Su, C.-C.; Shao, S.-C.; Sung, S.-F.; Lin, S.-J.; Yang, Y.-H.K.; Lai, E.C.-C. Taiwan's National Health Insurance Research Database: Past and future. Clin. Epidemiol. 2019, 11, 349. [CrossRef]

12. Lin, L.-Y.; Warren-Gash, C.; Smeeth, L.; Chen, P.-C. Data resource profile: The National Health Insurance Research Database (NHIRD). Epidemiol. Health 2018, 40, e2018062. [CrossRef]

13. Tonelli, M.; Wiebe, N.; Fortin, M.; Guthrie, B.; Hemmelgarn, B.R.; James, M.T.; Klarenbach, S.W.; Lewanczuk, R.; Manns, B.J.; Ronksley, P. Methods for identifying 30 chronic conditions: Application to administrative data. BMC Med. Inform. Decis. Mak. 2015, 15, 31 .

14. Piette, J.D.; Kerr, E.A. The impact of comorbid chronic conditions on diabetes care. Diabetes Care 2006, 29, 725-731. [CrossRef] [PubMed]

15. Redelmeier, D.A.; Tan, S.H.; Booth, G.L. The treatment of unrelated disorders in patients with chronic medical diseases. N. Engl. J. Med. 1998, 338, 1516-1520. [CrossRef] [PubMed]

16. Tonelli, M.; Wiebe, N.; Guthrie, B.; James, M.T.; Quan, H.; Fortin, M.; Klarenbach, S.W.; Sargious, P.; Straus, S.; Lewanczuk, R. Comorbidity as a driver of adverse outcomes in people with chronic kidney disease. Kidney Int. 2015, 88, 859-866. [CrossRef]

17. Deyo, R.A.; Cherkin, D.C.; Ciol, M.A. Adapting a clinical comorbidity index for use with ICD-9-CM administrative databases. J. Clin. Epidemiol. 1992, 45, 613-619. [CrossRef]

18. Ridgeway, G.; McCaffrey, D.; Morral, A.; Burgette, L.; Griffin, B.A. Toolkit for Weighting and Analysis of Nonequivalent Groups: A Tutorial for the Twang Package; RAND Corporation: Santa Monica, CA, USA, 2017.

19. Liu, P.; Quinn, R.R.; Karim, M.E.; Bello, A.; Tam-Tham, H.; Weaver, R.; Ronksley, P.E.; Quan, H.; Strippoli, G.F.; Manns, B. Nephrology consultation and mortality in people with stage 4 chronic kidney disease: A population-based study. CMAJ 2019, 191, E274-E282. [CrossRef] [PubMed]

20. Liu, P.; Quinn, R.R.; Cortese, G.; Mahsin, M.; James, M.T.; Ronksley, P.E.; Quan, H.; Manns, B.; Hemmelgarn, B.R.; Tonelli, M. Nephrology consultation and kidney failure in people with stage 4 chronic kidney disease: A population-based cohort study. $J$. Nephrol. 2021, 34, 1225-1234. [CrossRef]

21. Austin, P.C. An introduction to propensity score methods for reducing the effects of confounding in observational studies. Multivar. Behav. Res. 2011, 46, 399-424. [CrossRef] [PubMed]

22. Levin, A.; Stevens, P.E.; Bilous, R.W.; Coresh, J.; De Francisco, A.L.M.; De Jong, P.E.; Griffith, K.E.; Hemmelgarn, B.R.; Iseki, K.; Lamb, E. J Kidney Disease: Improving Global Outcomes (KDIGO) CKD Work Group. KDIGO 2012 clinical practice guideline for the evaluation and management of chronic kidney disease. Kidney Int. Suppl. 2013, 3, 1-150.

23. Iwata, Y.; Okushima, H.; Takatsuka, T.; Yoshimura, D.; Kawamura, T.; Iio, R.; Ueda, Y.; Shoji, T.; Hayashi, T.; Isaka, Y. Duration of predialysis nephrological care and mortality after dialysis initiation. Clin. Exp. Nephrol. 2020, 24, 705-714. [PubMed]

24. Salman, B.; Tahir, M.; Qureshi, R.; Dhrolia, M.F.; Ahmad, A.; Imtiaz, S. Factor causing late referral of CKD patients to nephrology care. Arch. Ren. Dis. Manag. 2017, 3, 26-29. [CrossRef] 